A N N A L E S Annales de Bretagne et des Pays de l'Ouest

\title{
Bretagne, la nation invisible (1750-1950)
}

\section{Yvon Tranvouez}

\section{OpenEdition}

\section{Journals}

Édition électronique

URL : http://journals.openedition.org/abpo/2818

DOI : $10.4000 / a b p o .2818$

ISBN : 978-2-7535-3513-8

ISSN : 2108-6443

\section{Éditeur}

Presses universitaires de Rennes

Édition imprimée

Date de publication : 30 juin 2014

ISBN : 978-2-7535-3511-4

ISSN : 0399-0826

Référence électronique

Yvon Tranvouez, "Bretagne, la nation invisible (1750-1950) 》, Annales de Bretagne et des Pays de l'Ouest [En ligne], 121-2 | 2014, mis en ligne le 30 juin 2014, consulté le 23 septembre 2020. URL : http:// journals.openedition.org/abpo/2818; DOI : https://doi.org/10.4000/abpo.2818 
l'une des régions les plus durement touchées. Ce constat, justifié par la grande part de ruraux affectés à l'infanterie et par une démographie dynamique fortement pourvoyeuse de mobilisés, a suscité de nombreuses récupérations dans une région où le rapport à la mort est important. L'inflation du chiffre des morts, scellé dans la pierre de Sainte-Anne-d'Auray à 240000 et parfois gonflé jusqu'à 300000 , légitime les revendications des uns et des autres et l'idée d'une dette sacrée contractée par la France. Ce rappel au sang versé justifie la volonté de reconnaissance régionale d'une Bretagne catholique affichée par l'Église, celle d'une intégration nationale exprimée par les républicains ou le séparatisme revendiqué par les mouvements nationalistes. Ce n'est pas le moindre atout de cet ouvrage, ni le moins courageux, que d'en finir avec ce fantasme doloriste, victimaire et identitaire d'une Bretagne sacrifiée, vision reconstruite et légendaire aux visées propagandistes encore très présente malgré les progrès de la recherche. Au final, cet ouvrage de grande qualité, aussi bien sur la forme que sur le fond, comble un vide et apporte un éclairage intelligent et très accessible intégrant les recherches historiques les plus récentes. Si l'on nous permet de célébrer tout de même un petit particularisme breton, saluons donc la parution de ce livre qui distinguera dans les rayonnages le sérieux et la qualité de l'historiographie bretonne sur le sujet.

Ronan RICHARD

GEMIE, Sharif, Bretagne, la nation invisible (1750-1950), Spézet, Coop Breizh, 2013, $328 \mathrm{p}$.

Il y a tellement peu de traductions de l'historiographie anglo-saxonne, chacun étant aujourd'hui supposé lire couramment l'anglais, qu'on ne peut que se réjouir de l'initiative mettant opportunément à la disposition du public de langue française cet ouvrage sur la Bretagne, écrit par un universitaire gallois. Tout au plus peut-on regretter que l'opération, sans doute un peu précipitée, n'ait pas fait l'objet d'un ultime examen qui aurait permis de la débarrasser de quelques distractions factuelles, de quelques lourdeurs stylistiques, ou d'un certain nombre de coquilles que le lecteur est obligé, selon la formule consacrée, de corriger lui-même.

Le titre intrigue. Pourquoi donc la Bretagne serait-elle une nation " invisible "? Parce qu'on la cache? Parce qu'elle se cache? Les deux, finit-on par comprendre. En gros, le nationalisme breton s'est trouvé doublement empêché, contrarié dès le XIXe siècle par la politique jacobine des gouvernements français et délégitimé au milieu du $\mathrm{Xx}^{\mathrm{e}}$ par le dévoiement fasciste de ses éléments les plus radicaux. Il en a été réduit, après 1945, à se recomposer comme une simple identité sociale et culturelle, rejoignant ainsi aujourd'hui le courant du "néonationalisme ", au sein de ce qu'il est convenu d'appeler les minorités nationales. Mais dans la variante soft : celle qui n'éprouve plus le besoin de créer des frontières ni donc d'accéder au pouvoir d'État, à la différence de la variante hard, type flamand, qui y prétend encore. "Les enfants de Mordrel, conclut l'auteur, ont, dans leur très grande majorité, rejeté son déterminisme de la terre et du sang pour créer quelque chose de plus subtil, de mieux adapté à l'échelle humaine : ils habitent une nation invisible " (p. 292).

Avant de s'achever sur ce puissant raccourci, le livre entraîne le lecteur dans un curieux parcours, parfois déconcertant, alternant vastes panoramas et observations de détail, à travers deux siècles dont les bornes temporelles annoncées dans le titre indiquent un cadre général plus qu'un programme exhaustif ou un itinéraire chronologique. Le premier chapitre, largement nourri des travaux de 
Jean-Yves Guiomar, rappelle l'enjeu du mythe celte dans la partition nationale française au XIX ${ }^{\mathrm{e}}$ siècle, et comment la pensée bretoniste a tendu à isoler la singularité celtique de l'héritage breton, repoussant l'identité française vers la romanité. Sharif Gemie insiste ensuite, à juste titre, sur la fracture induite en Bretagne par la Révolution française, le long d'une ligne essentiellement religieuse : c'est en effet la profondeur de cette cassure qui explique, dans la longue durée du XIX et du premier $\mathrm{xx}^{\mathrm{e}}$ siècle, l'impossible réconciliation bretonne de la gauche et de la droite, des bleus et des blancs. Le troisième chapitre, très stimulant, montre comment le concept d'orientalisme, emprunté à Edward Saïd, peut être appliqué aux discours dominants tenus sur la Bretagne au XIX ${ }^{\mathrm{e}}$ siècle. Renvoyant une image ambivalente d'archaïsme et d'authenticité, et distinguant la "vraie " Bretagne, bretonnante, de l'autre, " débretonnisée " selon le mot de Flaubert, ces stéréotypes favoriseront, à terme, une vision coloniale de la région.

La suite donne la priorité aux problèmes proprement politiques. On voit, dans le quatrième chapitre, comment les vaincus de la Révolution française, les blancs, s'emparent logiquement de la cause bretonne, étroitement associée à une mémoire enchantée de la chouannerie et dès lors interdite, en quelque sorte, aux bleus. Cette captation n'est cependant pas purement réactionnaire : en écho notamment aux travaux de Michel Lagrée, l'auteur rappelle qu'au tournant du XIX et du Xxe siècle, l'aristocratie et le clergé apparaissent comme les promoteurs d'une modernité catholique alternative à la républicaine. Mais surtout, ce n'est qu'une captation : la cause est bretonne par accident et non par destination. Les deux chapitres qui suivent inversent le point de vue en se plaçant du côté de l'État. Très fouillé, mobilisant abondamment des sources primaires, le cinquième étudie particulièrement le révélateur que constituent les fêtes politiques sous la Monarchie de Juillet et la Seconde République, tandis que le sixième, plus aérien, s'attache à la politique d'intégration nationale menée par le Second Empire puis la Troisième République (mais, contrairement à ce que le titre annonce généreusement, le $\mathrm{xx}^{\mathrm{e}}$ siècle est peu abordé). De ce triptyque, il ressort que, au moins jusqu'en 1914, la revendication bretonne n'est jamais que le visage conjoncturel d'une protestation d'une autre origine, qu'elle soit politique, sociale ou religieuse. Ainsi, quand la République est bonne fille, l'Église n'éprouve pas le besoin de faire local; mais quand Émile Combes paraît, la défense religieuse s'hypostasie en défense de la Bretagne. Feiz ha Breiz est moins une pétition de principe qu'une arme de circonstance. En somme, le catholicisme breton n'est qu'une déclinaison conjoncturelle de la Bretagne catholique, comme la démocratie bretonne de la Bretagne démocratique : l'isolement de l'abbé Perrot rejoint celui d'Émile Masson.

La fin de l'ouvrage s'attache à la configuration institutionnelle du mouvement breton, en distinguant logiquement régionalisme et nationalisme. Sharif Gemie rappelle dans le septième chapitre que le XIX ${ }^{\mathrm{e}}$ siècle est régionaliste, avec l'Association bretonne (née en 1843, interdite en 1859, ressuscitée en 1873), très conservatrice, l'Union régionaliste bretonne (1898) qui l'est à peine moins, le Bleun-Brug (1905), version catholique du mouvement breton, et la Fédération régionaliste bretonne (1911), dissidence de l'URB et première variante bleue de l'emsav. L'année 1911 voit aussi la fondation de l'éphémère et confidentiel Parti nationaliste breton. Mais c'est au lendemain de la Grande Guerre que le nationalisme se développe, porté par une nouvelle génération autour de Breiz Atao et de ses principales déclinaisons successives en Parti autonomiste breton (1927) et Parti national breton (1931), le tout-venant, dans le contexte de la crise des années trente, déborder le régionalisme par la droite. Le huitième et dernier chapitre, qui porte sur la période de l'Occupation, souligne l'effet ravageur, pour le mouvement breton, de l'engagement collaborationniste de ses représentants les plus exaltés, qui sont aussi les plus bruyants, et donc les plus 
visibles. La Bretagne s'en retrouve condamnée, de facto, à demeurer politiquement invisible, ou pour le dire dans le langage de l'entre-deux-guerres, à n'être pas plus qu'une petite patrie - purement décorative - dans la grande patrie française.

Tout cela n'est pas neuf, évidemment, mais on saura gré à l'auteur d'avoir proposé une vision d'ensemble de la question nationale bretonne, et non pas seulement une histoire du mouvement breton. L'ouvrage étant paru au Royaume-Uni en 2007, et ayant été écrit pour l'essentiel au début des années 2000, on ne s'étonnera pas qu'il ne fasse pas mention de travaux importants publiés depuis, comme ceux de David Bensoussan ou de Laurent Le Gall par exemple. On appréciera, en revanche, les nombreuses références à des recherches anglo-saxonnes que les historiens hexagonaux ne suivent pas toujours avec l'attention qu'il faudrait - et je le dis d'autant plus simplement que je suis le premier à devoir confesser cette insuffisance.

Yvon TRANVOUEZ

THomAs, Mannaig (dir.), La Bretagne Linguistique, n 17, Brest, CRBC, 2012, 286 p.

Cette livraison de La Bretagne Linguistique réunit les communications présentées lors des journées d'études organisées à Brest en 2010-2011 par le GRELB (Groupe de recherche sur l'économie linguistique de la Bretagne). Elle donne un bon aperçu de l'actualité de la recherche des universitaires brestois - ainsi que de chercheurs issus d'autres universités - portant sur des sources en langue bretonne, dans une perspective interdisciplinaire.

Les cinq premiers articles ont pour point commun le dictionnaire breton-français de Coëtanlem, dont plusieurs pages sont reproduites en couleur dans le volume. Acheté par la ville de Brest et conservé au CRBC, ce dictionnaire manuscrit de huit volumes totalise plus de 8300 pages. Il a été entièrement numérisé en 2008 et est désormais accessible sur le site Hermine [http://www.hermine.org]. Compilé par l'érudit Pierre Joseph Jean de Coëtanlem de Rostiviec (1749-1827), il reprend, commente et complète les principaux auteurs de dictionnaires bretons des $\mathrm{XVII}^{\mathrm{e}}$ et $\mathrm{XVIII}^{\mathrm{e}}$ siècles : Julien Maunoir (1659), Grégoire de Rostrenen (1732) et surtout Dom Louis Le Pelletier (1752). La version définitive du manuscrit a été mise en forme entre 1808 au plus tôt et 1820 au plus tard, même si le travail a vraisemblablement commencé au cours de la Révolution française. Le dictionnaire de Coëtanlem constitue donc, comme le suggère Ronan Calvez dans l'article introductif du volume, " la dernière somme encyclopédique - dans le sens des Lumières - du breton " (p. 15). L'objet du symposium organisé en décembre 2010 a été de mettre en valeur cette source longtemps ignorée des chercheurs. On peut toutefois regretter que ce salutaire travail de réhabilitation ne soit pas précédé par une présentation biographique approfondie de l'auteur : on ne parle en effet de la vie de Coëtanlem que par allusions, laissant au lecteur le soin de se reporter à la maigre littérature scientifique existant sur le sujet, à savoir deux courts articles de Louis Dujardin et François Falc'hun publiés en 1948 dans la Nouvelle Revue de Bretagne.

Dans un article très complet intitulé " Les dictionnaires bretons, une source pour l'ethnographie " (p. 25-64), Fañch Postic se concentre sur les pratiques calendaires liées aux quêtes des étrennes de fin d'année, sur lesquelles il travaille depuis plusieurs décennies. Il revient sur les attestations écrites en Bretagne et dans d'autres aires culturelles européennes et américaines en mettant en parallèle les informations contenues dans les dictionnaires avec de nombreuses sources depuis le $\mathrm{Xv}^{\mathrm{e}}$ siècle. En lien avec la pratique de cet eginane, un terme dont l'étymologie a suscité de 